\title{
An On-Chip Superconducting Kinetic Inductance Fourier Transform Spectrometer for Millimeter-Wave Astronomy
}

\author{
F. B. Faramarzi ${ }^{1,2} \cdot$ P. Mauskopf ${ }^{1,2} \cdot$ S. Gordon ${ }^{2,3} \cdot$ G. Che $^{4} \cdot$ P. Day ${ }^{5}$.

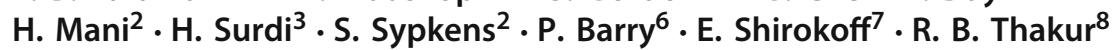

Received: 26 August 2019 / Accepted: 2 December 2019 / Published online: 7 January 2020

(c) Springer Science+Business Media, LLC, part of Springer Nature 2020

\begin{abstract}
An on-chip FTS consists of two waveguides coupled to long superconducting transmission lines (STLs) $(\sim 620 \mathrm{~mm})$ using two coupling probes. The signal propagating on one of the STLs is phase-shifted with respect to the other line with a bias current that affects the nonlinear dependence of kinetic inductance, $\mathscr{L}_{k}(I)$ of the STL material. Here we describe the design and simulation of a superconducting on-chip FTS coupled to a dual polarization W-band (90-110 GHz) waveguide. These devices have applications in ground-based and space-based millimeter-wave spectral surveys.
\end{abstract}

Keywords Fourier transform spectrometer $\cdot$ Kinetic inductance $\cdot$ Superconducting transmission lines $\cdot$ Millimeter-wave astronomy

\section{Introduction}

Interferometric techniques are widely used in astronomy for imaging and spectroscopy purposes [1-6]. The measured signal using an interferometer is in the conjugate space to focal plane imaging. Therefore, the signal from any one element of an

$凶 \quad$ F. B. Faramarzi

ffarama1@asu.edu

1 Department of Physics, Arizona State University, Tempe, AZ 85281, USA

2 School of Earth and Space Exploration, Arizona State University, Tempe, AZ 85281, USA

3 School of Electrical, Computer, and Energy Engineering, Arizona State University, Tempe, AZ 85281, USA

4 Georgia Tech Research Institute, Atlanta, GA 30318, USA

5 Jet Propulsion Laboratory, Pasadena, CA 91109, USA

6 Argonne National Laboratory, Lemont, IL 60439, USA

7 The Department of Physics, The University of Chicago, Chicago, IL 60637, USA

8 California Institute of Technology, Pasadena, CA 91125, USA 
imaging instrument is spread out among all the elements of the spectrometer [7]. For millimeter/sub-millimeter astronomy, imaging interferometers can be used for measurements of cosmic microwave background (CMB) fluctuations and spectral distortions [8-10], line intensity mapping of spectral lines [11-13], and novel on-chip calibration technology for sub-millimeter focal planes. Some current experiments that use Fourier transform spectrometers are HERSCHEL [14], SCUBA [15] and OLIMPO [16]. The average size of such FTS instruments is one meter, and they have a frequency resolution ranging from $150 \mathrm{MHz}$ to $6 \mathrm{GHz}$. Other experiments such as DESHIMA [17] and Superspec [18] use on-chip filter bank technology with spectral resolutions of 200-1000.

In this work, we present an on-chip superconducting kinetic inductance Fourier transform spectrometer (FTS) for millimeter and sub-millimeter observational cosmology and astrophysics. This design is very compact with chip dimension of about one inch by quarter of an inch and has spectral resolution better than 1000. Our superconducting FTS works based on the principle of modulating the phase of the incident radiation by controlling the kinetic inductance of its transmission lines[19]. In Sect. 2, we describe the operating principles of such on-chip FTS in detail and give an estimation of phase shift and spectral resolution of the device in the W-band. In Sect. 3, we go over a circuit design based on parameters explained in Sect. 2. Section. 4 explains the electromagnetic coupling to the on-chip FTS and results we obtained from simulations. Our plans for testing the said devices and the measurement setup are mentioned in Sect. 5. Lastly, in Sect. 6 we describe our future test plans and circuit design for an antenna and detector coupling of the FTS.

\section{Operating Principles}

The operating principle of an on-chip FTS is very similar to a Michelson interferometer in that an incident beam splits into two by passing through a beam splitter. Each beam is then reflected from a mirror and recombined in the detector. Changing the path length of one of the beams by moving one mirror and keeping the other fixed will cause a phase shift in the beam reflected from the movable mirror, which will interfere constructively or destructively with the other beam [20]. In a similar manner, the optical power from the sky is coupled to the FTS using a broadband antenna and then split into two beams using a $180^{\circ}$ hybrid and recombined in two detectors, as shown in Fig. 1.

An on-chip superconducting FTS consists of two superconducting transmission lines (STLs). In designing these devices, we take advantage of the nonlinear behavior of the kinetic inductance of superconductors [21]. When AC biased, superconductors show an additional inductance to their geometric inductance due to the finite mass of Cooper pairs [22]. From BCS theory, kinetic inductance is given by [23,24]:

$$
L_{k}=\frac{\hbar \rho_{n}}{\pi \Delta t}
$$



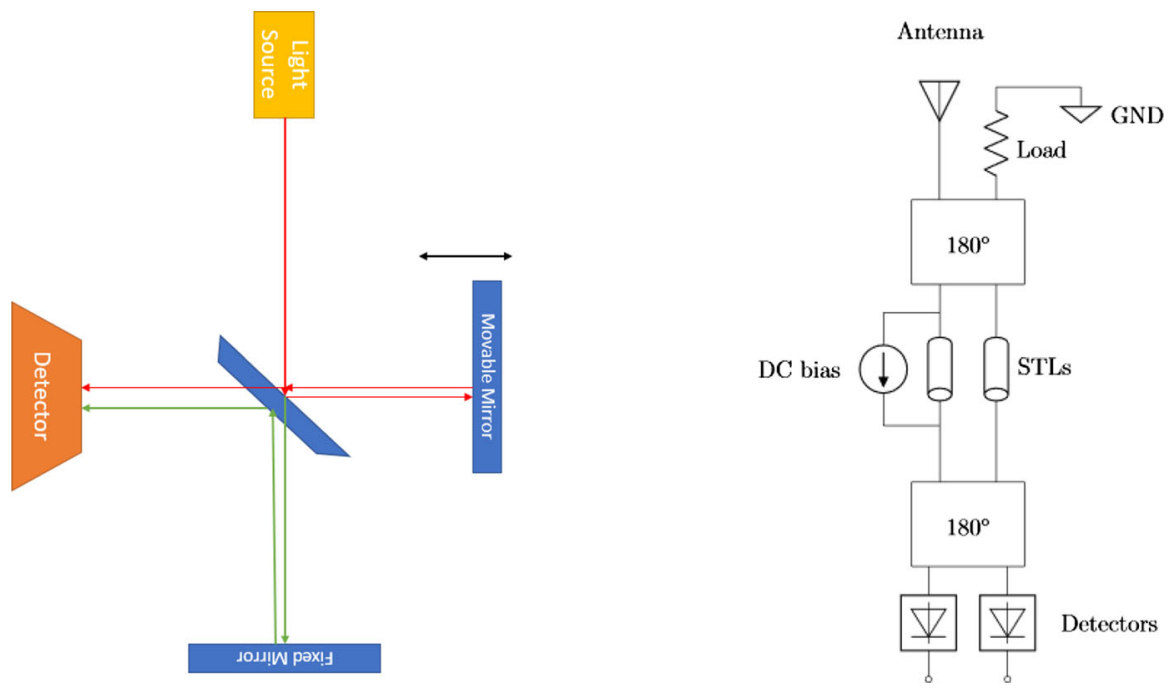

Fig. 1 left A typical Michelson interferometer. right The circuit diagram of an on-chip FTS (color figure online)

where $\hbar$ is Planck's constant, $\rho_{n}$ is the normal resistivity of the film, $\Delta$ is the band gap of the superconductor and $t$ is the thickness of the film. Within a certain regime set by the characteristic current $I_{*}$, the kinetic inductance of the superconductor varies nonlinearly with bias current $I$. This relation is given by [22]

$$
L_{k}(I) \simeq L_{K}(0)\left[1+\left(\frac{I}{I_{*}}\right)^{2}\right]
$$

where $L_{k}(0)$ is the kinetic inductance at $I=0$.

Therefore, the characteristic impedance of a STL is given by

$$
Z_{0}=\sqrt{\frac{\mathscr{L}_{k}(I)+\mathscr{L}_{g}}{\mathscr{C}}}
$$

with $\mathscr{C}$ being the capacitance per unit length of the STL. A signal through a STL travels with the speed of $v_{p}(I)=1 / \sqrt{\mathscr{L}_{\text {tot }} \mathscr{C}}$, with $\mathscr{L}_{\text {tot }}$ being the sum of kinetic and geometric inductances per unit length of the STL $\left(\mathscr{L}_{k}+\mathscr{L}_{g}\right)$ and $\mathscr{C}$ being the total capacitance per unit length of the STL. This gives a phase shift of

$$
\Delta \phi(I)=\frac{\omega l}{v_{p}(0)}\left(1-\frac{v_{p}(0)}{v_{p}(I)}\right)
$$

where $\omega$ is the angular frequency and $l$ is the length of the STL. Therefore, we can make an interferogram by splitting the incident signal between two STLs, modulating the current $I$ with a DC bias after recombining the signals in the detector. For a $620 \mathrm{~mm}$ long transmission line and nonlinear kinetic inductance parameters corresponding 


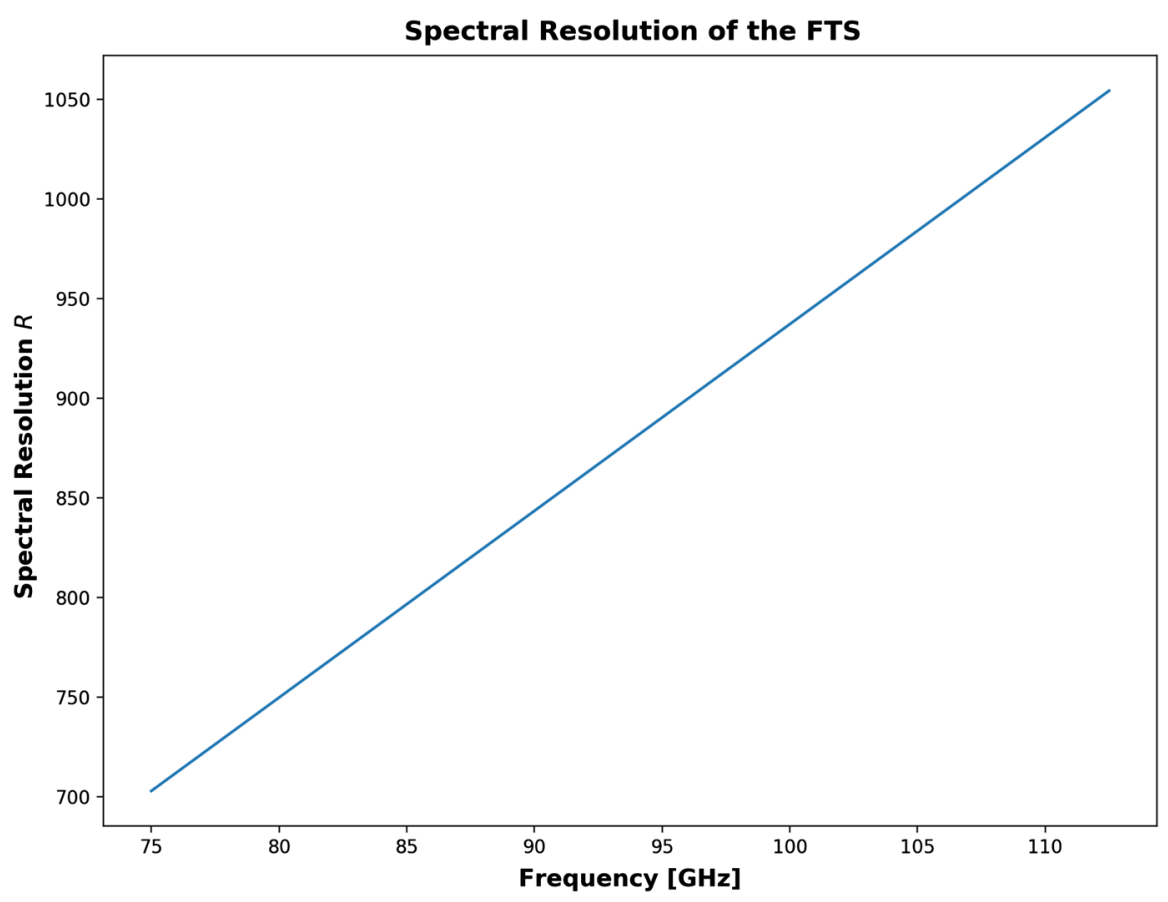

Fig. 2 Spectral resolution of an on-chip FTS in the W-band regime (color figure online)

to $\mathrm{NbTiN}$ of $I_{*}=0.12 \mathrm{~mA}$ [28] and $\hbar \rho_{n} / \pi \Delta_{0}=0.28 \mathrm{nH} . \mathrm{nm}$ [27], we estimate a maximum phase shift of 1767 rad corresponding to a frequency resolution of $\Delta v \sim 100$ $\mathrm{MHz}$ at $90 \mathrm{GHz}$. The spectral resolution of such a device as a function of frequency is shown in Fig. 2. We can achieve higher spectral resolution by increasing the inductance or the capacitance of the STLs.

\section{Circuit Design}

For the STLs, we have designed a $620 \mathrm{~mm}$ long meandered inverted microstrip line geometry where the strip line is a thin film of superconducting material ( $\mathrm{TiN}, \mathrm{NbN}$, NbTiN, etc.) [25] deposited on a high-resistivity silicon substrate. The chip containing the STLs sits on a package in an inverted orientation. Therefore, the ground of the microstrip line is provided by the ground plate of the chip package (Fig. 3).

\section{Electromagnetic Coupling}

At this stage, we have used two pairs of superconducting probes with perpendicular polarization, as shown in Fig. 4. Ideally, we expect 50\% of incident radiation gets absorbed by each pair of probes through the silicon substrate. In order to get maximum coupling, we optimized the coupling efficiency of the probes by changing the 

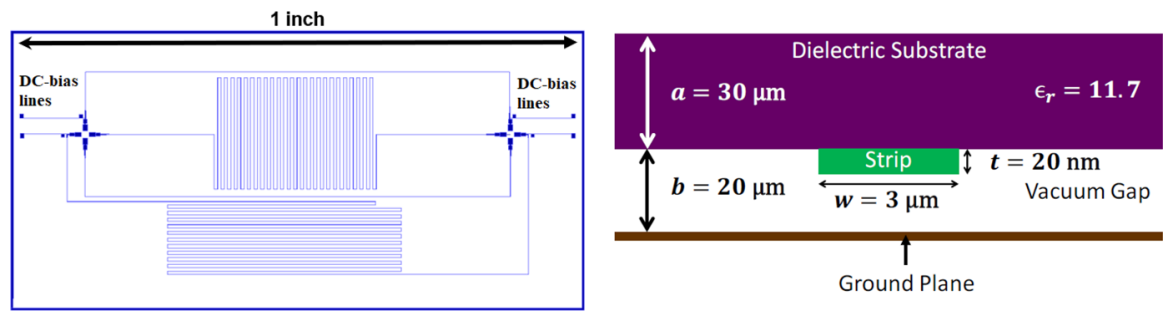

Fig. 3 left A mask design of an on-chip FTS with the DC bias lines to the right and left of the probes. The STLs are in the form of meander geometry. right The inverted microstrip line geometry used to design the STLs. The superconducting film is deposited on a silicon substrate, and there exists a $20 \mu \mathrm{m}$ gap between the substrate and the ground plane (color figure online)
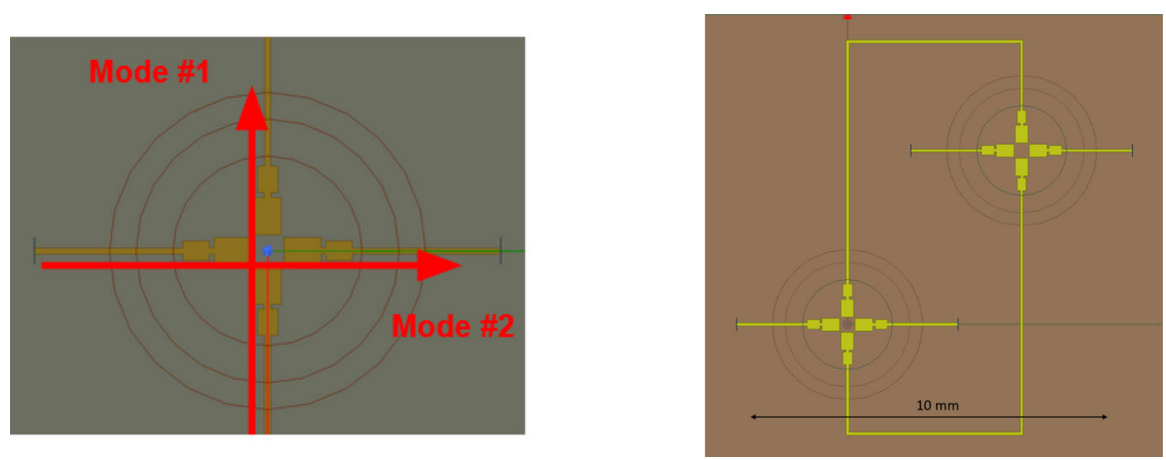

Fig. 4 left Two perpendicular modes of the waveguide (top view). right Transmission simulation setup of the input and output waveguides for one of the modes(top view). The black circles show the locations of the waveguide and the choke (color figure online)

thickness of the silicon substrate and simulated it in Ansys HFSS software [26]. Figure 5 shows the coupling efficiency versus frequency for different silicon thicknesses. We expect optimum efficiency across the W-band for silicon thickness in the range $25 \mu \mathrm{m} \leq a \leq 40 \mu \mathrm{m}$. In order to achieve this, we only etch the area under the probes to make the FTS chip less brittle. The plot in Fig. 4 also gives us an idea of the tolerance of the etch process.

We also need to check the transmission of one of the modes through the waveguides by terminating the opposite mode probes. As is shown in Fig. 4, the scattering parameters, $S_{21}$, for various modes show that in most of the band $70 \%$ of the power is transmitted from one waveguide port to the other, while the isolation from the other ports is better than $-30 \mathrm{~dB}$ over the entire band (color figure online) (Fig. 6).

In Fig. 4, there is almost $100 \%$ transmission between waveguides through the connected probes for mode number one between 90 and $110 \mathrm{GHz}$. As expected, the terminated probes have zero transmission. The black and blue curves show the cross talk between the modes, which are also suppressed. 


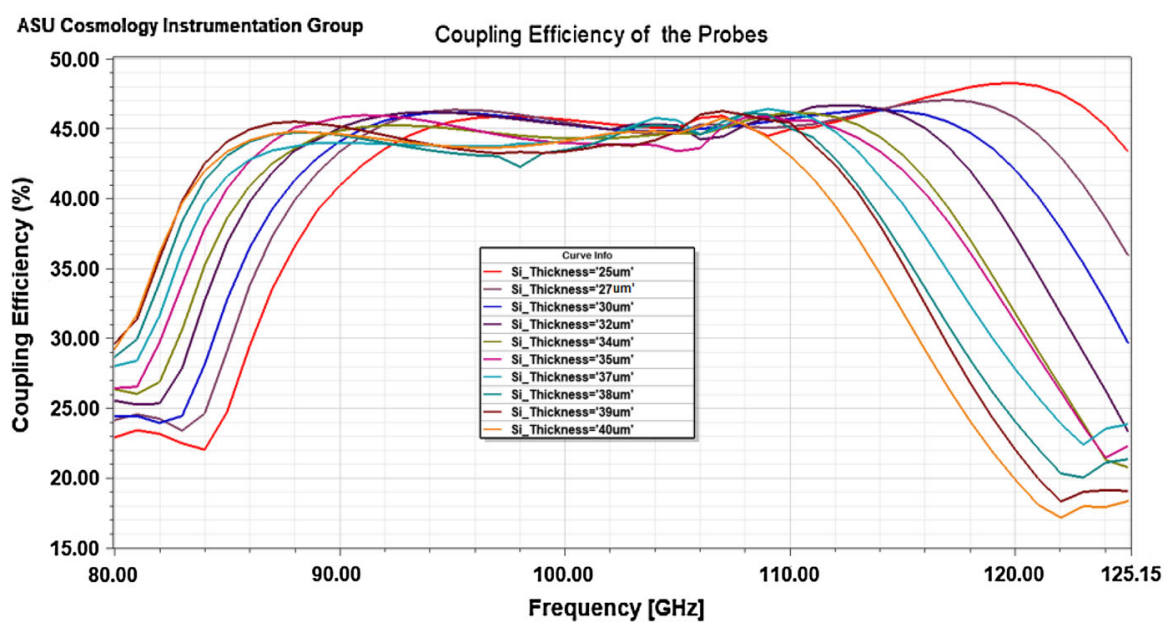

Fig. 5 Plot of electromagnetic coupling efficiency of the probes in the W-band regime. The transmission coefficient, $\mathrm{S}_{21}$, from port 1 to port 2 in band is approximately $-3.5 \mathrm{~dB}$ or $45 \%$. All of the loss is through leakage out of the gap between the incoming waveguide and the chip and is absorbed by matched impedance absorbers on the walls of the silicon in the simulation (color figure online)

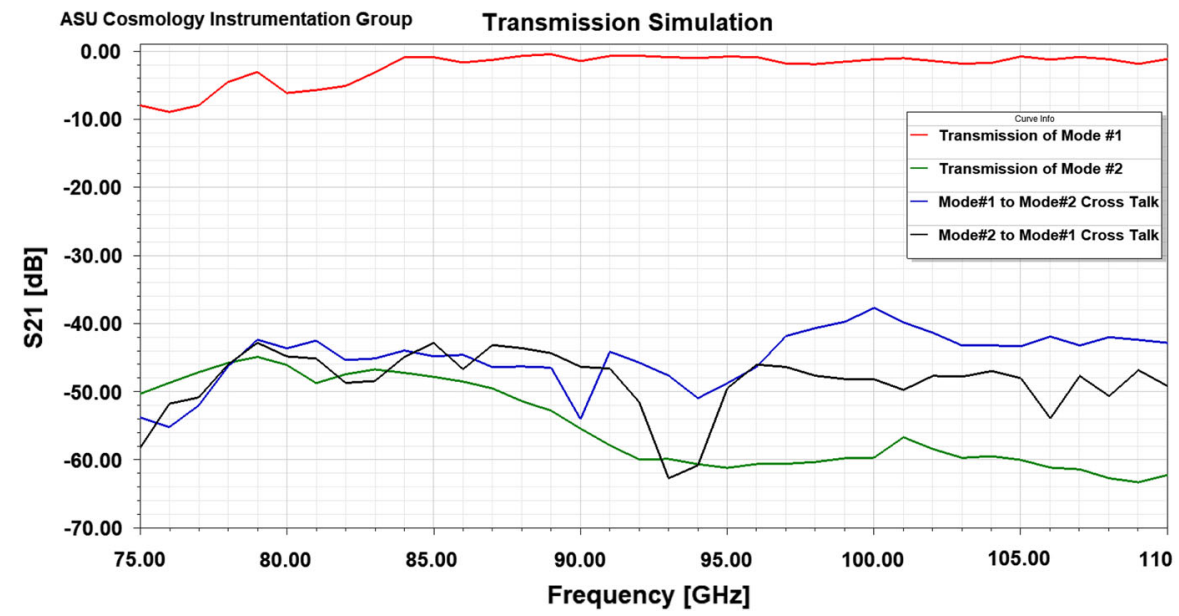

Fig. 6 Red curve is transmission simulation of mode one through a microstrip line. Green curve is transmission simulation of terminated port number two. Blue and black curves are the cross talk between modes one and (color figure online) two

\section{Test Setup}

The cryogenic W-band test setup consists of the device at the 4K stage of a GM cryocooler with a custom-made waveguide feedthrough, which has a thermal break and vacuum window design to deliver the W-band signal to the device based on [29] and [30]. We use a vector network analyzer (VNA) with W-band extenders to perform the measurements. To DC-bias the STLs, we use bias tees mounted on the chip package 
and sweep the current from 0 to $I_{*}$ while providing a $90 \mathrm{GHz}$ tone to measure the phase shift $\Delta \phi$.

\section{Future Work}

The primary plan is to measure the phase shift $\Delta \phi$ of $\mathrm{NbN}$ and NbTiN chips fabricated at ASU and Caltech. After measuring the phase shifts and comparing them to the expected values, [21] the next stage will be to couple the FTS to a wideband superconducting antenna and kinetic inductance detectors [31], which are integrated on the same chip. Lastly, we intend to make an array of superconducting antenna and detector-coupled FTSs for future interferometry experiments.

Acknowledgements I would like to thank Ms. Emily Lunde and Mr. Adrian Sinclair for their helpful discussions and support.

\section{References}

1. L. Earle, P. Ade, J. Aguirre, R. Aikin, J. Battle, J. Bock, C.M. Bradford, M. Dragovan, L. Duband, J. Glenn, G. Griffin, V. Hristov, P. Maloney, H. Matsuhara, B. Naylor, H. Nguyen, M. Yun, J. Zmuidzinas, Proc. SPIE 6275, 627510 (2006). https://doi.org/10.1117/12.672309

2. B.J. Naylor, P.A.R. Ade, J.J. Bock, C.M. Bradford, M. Dragovan, L. Duband, L. Earle, J. Glenn, H. Matsuhara, H. Nguyen, M. Yun, J. Zmuidzinas, Proc. SPIE 4855, 239 (2003). https://doi.org/10.1117/ 12.459419

3. D.J. Fixsen et al., AJ 508, 123 (1998). https://doi.org/10.1086/306383

4. T.R. Greve et al., MNRAS 359, 1165 (2005). https://doi.org/10.1111/j.1365-2966.2005.08979.x

5. S. Aalto et al., A \& A 381, 783 (2002). https://doi.org/10.1051/0004-6361:20011514

6. C.M. Bradford et al., AAS Meeting 205, 10 (2004)

7. J. Zmuidzinas, J. Opt. Soc. Am. A 2, 218 (2003). https://doi.org/10.1364/JOSAA.20.000218

8. C. Kouveliotou, E. Agol, N. Batalha, J. Bean, M. Bentz, N. Cornish, A. Dressler, E. Figueroa-Feliciano, S. Gaudi, O. Guyon, D. Hartmann, J. Kalirai, M. Niemack, F. Ozel, C. Reynolds, A. Roberge, K. S. A. Straughn, D. Weinberg, J. Zmuidzinas, (2014). arXiv e-prints, arXiv:1401.3741)

9. P. Serra, O. Dore, G. Lagache, ApJ 833, 153 (2016). https://doi.org/10.3847/1538-4357/833/2/153

10. A. Ghribi, B. Belier, F. Boussaha, E. Breelle, M. Piaf, S. Spinelh, A. Tartari, M. Zannoni, AIPC 1185, 506 (2009). https://doi.org/10.1063/1.3292390

11. E. Visbal, A. Loeb, J. Cosmol. Astro-Particle Phys. 2010, 016 (2010). https://doi.org/10.1088/1475$7516 / 2010 / 11 / 016$

12. Y. Gong, A. Cooray, M.G. Santos, ApJ 768, 130 (2013). https://doi.org/10.1088/0004-637X/768/2/ 130

13. C.M. Casey, D. Narayanan, A. Cooray, Phys. Rep. 45, 541 (2014). https://doi.org/10.1016/j.physrep. 2014.02.009

14. B.M. Swinyard et al., Mon. Notices R. Astron. Soc. 4, 3658 (2014). https://doi.org/10.1093/mnras/ stu409

15. D. Naylor et al., Proce. SPIE 5159, (2004). https://doi.org/10.1117/12.506395

16. A. Schillaci et al., AA A125, 565 (2014). https://doi.org/10.1051/0004-6361/201423631

17. A. Endo et al., J. Low Temp. Phys. 167, 341 (2012). https://doi.org/10.1007/s10909-012-0502-1

18. J. Wheeler and et al., Proc. SPIE 9914, 99143K9, https://doi.org/10.1117/12.2233798

19. G. Bordier, V.D. Cammilleri, B. Bélier, N. Bleurvacq, A. Ghribi, M. Piat, A. Tartari, M. Zannoni, J. Low Temp. Phys. 176, 663 (2014). https://doi.org/10.1007/s10909-014-1103-y

20. E. Hecht, Optics, 5th edn. (Pearson, 2016), ISBN-10: 0133977226

21. G. Che, Advancements in Kinetic Inductance Detector, Spectrometer, and Amplifier Technologies for Millimeter-Wave Astronomy, Ph.D. Dissertation, Arizona State University (2018) 
22. P. Mauskopf, IOP Publ. 130, 990 (2018). https://doi.org/10.1088/1538-3873/aabaf0

23. J. Bardeen, L.N. Cooper, J.R. Schrieffer, Phys. Rev. 106, 162-164 (1957). https://doi.org/10.1103/ PhysRev.106.162

24. M. Tinkham, Introduction to Superconductivity, 2nd edn. (Dover Publications, 2014), ISBN-10: 0486435032

25. R.B. Thakur et al., J. Low Temp. Phys. This Special Issue (2019)

26. ANSYS HFSS software, http://www.ansoft.com/products/hf/hfss/

27. Y. Shim, C. Tahan, IEEE J. Sel. Top. Quant. Electron. 21, 1-9 (2014). https://doi.org/10.1109/JSTQE. 2014.2358208

28. B. Eom, P. Day, H. LeDuc, J. Zmuidzinas, Nat. Phys. 8(Supplementary Information) (2012), https:// doi.org/10.1038/NPHYS2356

29. S. Melhuish, M. McCuloch, L. Piccirillo, C. Stott, Rev. Sci. Instrum. 87, 104706 (2016). https://doi. org/10.1063/1.4964475

30. G. Ediss, N. Horner, F. Johnson, D. Koller, A. Kerr, WR-10 Waveguide Vacuum Feedthrough for the ALMA Band-6 Cartridge, NRAO, (2005)

31. J. Meinke et al., J. Low Temp. Phys. This Special Issue (2019)

Publisher's Note Springer Nature remains neutral with regard to jurisdictional claims in published maps and institutional affiliations. 